\title{
Rilpivirine use in the Swiss HIV cohort study: a prospective cohort study
}

\author{
Delphine Sculier ${ }^{1 *}$, Angèle Gayet-Ageron ${ }^{2}$, Manuel Battegay ${ }^{3}$, Matthias Cavassini ${ }^{4}$, Jan Fehr ${ }^{5}$, Cedric Hirzel ${ }^{6}$, \\ Patrick Schmid ${ }^{7}$, Enos Bernasconi ${ }^{8}$, Alexandra Calmy ${ }^{1}$ and for the Swiss HIV Cohort Study
}

\begin{abstract}
Background: Rilpivirine is safe and effective in HIV-naïve patients with low baseline HIV-RNA or in switch strategy. It offers the advantages of few drug-drug interactions and a favourable toxicity profile. We aimed to determine the reasons for prescribing the rilpivirine (RPV)/tenofovir disoproxil (TDF)/emtricitabine (FTC) co-formulation within the Swiss HIV Cohort Study and to assess its effectiveness and safety over a 24 months period.

Methods: All individuals enrolled in the Swiss HIV Cohort Study who initiated a RPV/TDF/FTC co-formulation between April 2013 and March 2014 were included. Primary outcomes were the HIV-RNA viral load (copies/mL) and CD4 cell count (cells $/ \mathrm{mm}^{3}$ ) at 6, 12 and 24 months. Reasons for a switch to RPV/TDF/FTC were evaluated through a standardized questionnaire. We also assessed discontinuation and reasons for discontinuation of RPV/TDF/FTC until October 30, 2015.

Results: Of 644 individuals who started the RPV/TDF/FTC co-formulation, only $7.5 \%$ were treatment-naive. At 24 months, viral suppression (HIV-RNA <50 copies/mL) was achieved in 100\% and $96.7 \%$ of cART-naïve and cART-experienced patients respectively. The switch to RPV was mainly done for simplification (44.6\%) and to overcome central nervous system toxicity symptoms due to efavirenz (24\%). Six months after switch, $74.8 \%$ of patients reported an improvement of psycho-neurological symptoms with continued improvement at 12 months for almost $80 \%$. However, one quarter of patients reported a discontinuation of RPV/TDF/FTC on October 30, 2015 after a median time of 18.4 months. Reasons for discontinuation included physician decision (5.3\%) and side-effects (3.9\%) mainly related to the central nervous system and to renal toxicity.

Conclusion: The RPV/TDF/FTC co-formulation was safe and effective throughout 24 months of follow-up but barely prescribed for HIV-naïve patients. Despite excellent virological suppression among both treatment-naïve and-experienced patients, we observed a high rate of treatment discontinuation.
\end{abstract}

Keywords: HIV-1, Rilpivirine, First-line regimen, Treatment simplification, Virological response, Safety

\section{Background}

The combination of rilpivirine (RPV), tenofovir disoproxil (TDF) and emtricitabine (FTC) has demonstrated noninferior efficacy in randomized controlled trials compared to efavirenz (EFV)-based regimens among HIV-infected, treatment-naïve patients with a baseline viral load of less than $10^{5}$ copies/mL [1-4]. RPV/TDF/FTC has also demonstrated efficacy in interventional [5] and observational [6-11] studies among treatment-experienced virologically suppressed patients switching to RPV. Current strategies

\footnotetext{
* Correspondence: delphine.sculier@hcuge.ch

'Division of Infectious Diseases, University Hospital Geneva, Geneva, Switzerland

Full list of author information is available at the end of the article
}

with combined antiretroviral treatment (cART) focus on efficacy and safety in an aging HIV population with multiple comorbidities requiring additional treatments [12]. In this setting, RPV offers the advantages of few drug-drug interactions [13] and a favourable toxicity profile with a low incidence of grade $2-4$ side-effects $[14,15]$. In particular, fewer discontinuations due to central nervous system (CNS) adverse events in patients receiving RPV were reported compared to patients on EFV-based regimens [16]. Of note, EFV-related CNS toxicity has prompted its removal as a first-line regimen from several international guidelines in favour of integrase inhibitors, protease inhibitors [17] or RPV [18]. An improvement of lipid parameters was also observed with RPV compared 
to EFV or protease inhibitors, with a decrease in total and low-density lipoprotein cholesterol and triglycerides $[4,5]$.

RPV, co-formulated with TDF and FTC in a single tablet, was initially approved in Switzerland on April 1, 2013, for the treatment of HIV-naïve patients with a viral load less than $10^{5}$ copies $/ \mathrm{ml}$. Our experience suggested that the RPV/TDF/FTC co-formulation was used among virologically suppressed cART-experienced patients in a switch strategy, either to reduce adverse events due to the current regimen or for treatment simplification. We sought to evaluate the reasons for the prescription, as well as for discontinuation, of the RPV/TDF/FTC co-formulation in participants in the Swiss HIV Cohort Study (SHCS) after it entered the Swiss market. In addition, we assessed its effectiveness and safety at 6 (M6), 12 (M12) and 24 months (M24) post-initiation under routine clinical conditions.

\section{Methods}

\section{Study design and patient population}

We conducted a prospective analysis among all HIV-1 infected adults $\geq 18$ years old participating in the SHCS who received at least one dose of the RPV/TDF/FTC coformulation between April 1, 2013, and March 31, 2014. The SHCS is a multicentre prospective study established in 1988 and continuously enrolling HIV-infected individuals [19]. In brief, patients receive HIV care at one of the 7 outpatient clinics of the SHCS (Basel, Bern, Geneva, Lausanne, Lugano, St. Gallen, Zurich), at participating regional hospitals, or from collaborating private physicians. Follow-up visits are scheduled on a 6-monthly basis and include physical assessment, adherence check, review of medical conditions and drug prescriptions, as well as laboratory examinations [20]. Approximately $75 \%$ of all HIV infected patients on cART in Switzerland are followed within the SHCS network [21]. The scientific board of the SHCS approved this prospective analysis and all patients signed an informed consent form before enrolment in the SHCS.

\section{Variables}

Primary outcomes assessed treatment effectiveness through the HIV-RNA viral load (copies/mL) and CD4 cell count (cells $/ \mathrm{mm}^{3}$ ) measured at M6, M12 and M24 following initiation of the RPV/TDF/FTC co-formulation. We defined virological suppression as HIV-RNA $<50$ copies $/ \mathrm{mL}$. Secondary outcomes assessed treatment safety and used total cholesterol $(\mathrm{mmol} / \mathrm{L})$, high-density lipoprotein $(\mathrm{mmol} / \mathrm{L})$, triglycerides $(\mathrm{mmol} / \mathrm{L})$, alanine aminotransferase $(\mathrm{UI} / \mathrm{L})$, creatinine $(\mu \mathrm{mol} / \mathrm{L})$, and the estimated glomerular function rate (eGFR) $\left(\mathrm{ml} / \mathrm{min} / 1.73 \mathrm{~m}^{2}\right)$ calculated according to the Modification of Diet in Renal Disease equation, as well as body mass index $\left(\mathrm{kg} / \mathrm{m}^{2}\right)$. Sociodemographic and clinical data were prospectively collected as part of the SHCS 6- monthly assessments at the time of initiation of the RPV/ TDF/FTC co-formulation (referred to as baseline) and at M6, M12 and M24.

Reasons for switching to RPV/TDF/FTC were collected retrospectively. From January 1, 2015, onwards, the HIV Cohorts Data Exchange Protocol (HICDEP) coding [22] was used to document treatment switches in the SHCS database. However, before this date, treating physicians did not have the possibility to code regimen changes intended to simplify treatment. To obtain these data, we conducted a survey among treating physicians of all patients included in our study. We created a standardized questionnaire with a closed list of reasons for switching, which was first evaluated to assess its reliability among 6 physicians at the HIV unit of Geneva University Hospitals. The questionnaire was then sent to all physicians participating in the SHCS who had switched their patients to the RPV/TDF/FTC co-formulation. When physicians declared the reason for the switch as "toxicity, predominantly from CNS", they were asked to give the exact reasons from a list of CNS-specific symptoms: i) symptoms of depression; ii) sleep disturbances/insomnia; iii) abnormal dreams; iv) dizziness/vertigo; v) fatigue/tiredness; and vi) other. Physicians were also required to document CNS symptoms at M6 and M12 after the switch as "worsening", "stable", "improvement", "not available" or "other".

In addition, we assessed a cross-section of patients who had discontinued the RPV/TDF/FTC co-formulation within M24 post-treatment initiation in both the treatment-naïve and -experienced groups and the reasons for discontinuation according to the HICDEP coding registered in the SHCS database.

\section{Statistical analysis}

We differentiated two groups of patients: those who started $\mathrm{RPV} / \mathrm{TDF} / \mathrm{FTC}$ as a first-line regimen (cART-naïve patients) and those who switched from any cART regimen to RPV/TDF/FTC (cART-experienced patients).

We assessed if virological suppression were different across the 3 time-points (M6, M12 and M24) compared to baseline values among cART-experienced and among cART-naïve patients separately. To take into account the repetition of measurement in the same subject, we developed a generalized estimating equation using the binomial family, and exchangeable correlation structure. All models were adjusted for gender, age, history of AIDS event, HCV and HBV positivity. Then we used a linear multilevel model with a random effect on the patient to compare the evolution of the CD4 count across the 3 time-points compared to baseline values first among cART-experienced then among cART-naïve patients. We adjusted both models for the same variables described above plus the CD4 nadir $(<50,50-99,100-199,200-349,350-499$ and $>=500)$ and viral load $(<=$ or $>50$ copies $/ \mathrm{mL})$. For safety parameters, we 
performed linear multilevel models with a random effect on the patient to compare the evolution of each parameter across the 3 time-points compared to baseline values. Again we performed the analyses separately in cART-experienced then in CART-naïve patients, and adjusted for gender, age, history of AIDS event, HCV and HBV positivity. We repeated the three models assessing the evolution of lipids (one model for cholesterol, triglycerides and finally HDL-cholesterol) across the 3 time-points compared to baseline values in the subgroup of cART-experienced patients previously treated with 2 NRTIs and one PI.

$P$ values were calculated from generalized estimating equation (for viral load) and linear multilevel models (CD4, ALAT, creatinine, eGFR, cholesterol, triglycerides, HDLcholesterol and BMI) comparing data at M6, M12 and M24 to baseline among cART-naïve then among cARTexperienced patients, after adjustment for confounders described above.

Finally, we reported the proportions of cART-experienced and -naive patients who discontinued the RPV/TDF/FTC co-formulation before October 30, 2015, and described the reasons.

All $P$ values reported were two-sided and the level of significance was set at 0.05 . Statistical analyses were conducted in STATA software, version 14 (StataCorp LP, College Station, TX, USA).

\section{Results}

Baseline characteristics of patients initiating RPV/TDF/FTC co-formulation

Between April 1, 2013, and March 31, 2014, 644 HIVinfected patients enrolled in the SHCS started the new RPV/TDF/FTC co-formulation. Most were male (70\%; 451/ 644), Caucasian $(73.9 \% ; 476 / 644)$, and men who have sex with men (MSM) (47.7\%; 307/644). Mean duration of HIV infection was 11 years ( \pm standard deviation [SD]: 7.8) and mean age, 45.8 years ( \pm 11.0 years); mean $\mathrm{CD} 4$ cell count at baseline and mean CD4 nadir were $637( \pm 271)$ and 283 cells $/ \mathrm{mm}^{3}( \pm 186)$, respectively. Among the 644 patients, 48 (7.5\%) were cART-naive at initiation of the RPV/TDF/ FTC co-formulation, representing $10.5 \%$ of the total number of HIV-naïve patients enrolled in the SHCS and initiated cART during the same time period $(n=456)$. The baseline characteristics of patients initiating the co-formulation are shown in Table 1 and are presented for cART naïve and experienced patients. Among cART experienced patients, 44 (7.4\%) were not fully virologically suppressed (HIV-RNA > 50 copies/mL, mean HIV-RNA 16629 copies $/ \mathrm{mL}$ ) at the time of the switch.

\section{Reasons for a switch to RPV/TDF/FTC in CART-experienced patients}

The study questionnaire was sent to the SHCS treating physicians in November 2014. At that time, we had identified 598 patients who were switched to RPV/ TDF/FTC during the study period. Four patients were excluded as one did not receive the RPV/TDF/FTC coformulation and 3 were cART-naive according to the treating physicians. The non-response rate was 9.5\% (57/ 598 questionnaires). For these 57 patients, as well as two additional patients identified later and without a questionnaire, we retained the reason for the switch as registered in the SHCS database. The mean duration of cART treatment at the time of switch was 8.7 years (SD: \pm 5.9 ). Before switching, $47.8 \%$ (285/596) of cART-experienced patients were on a non-nucleoside reverse transcriptase inhibitor (NNRTI)-based regimen, $26.2 \%(156 / 596)$ were on a protease inhibitor-based regimen, and 9.7\% (58/596) were on an integrase inhibitor-based regimen. Of those on a NNRTI regimen, two thirds (192/285) were on EFV. Regimens before switch and reasons for switch are presented in Table 2. Treatment simplification (266/596; 44.6\%) and CNS toxicity $(143 / 596 ; 24.0 \%)$ were the two main reasons for switch.

CNS toxicity was the reason for the switch for 126 patients on an EFV-based regimen (65.6\%; 126/192) with a description of symptoms available for 123 patients (Table 3). Among these, $60.2 \%$ (74/123 patients) had more than one symptom, and a total of 197 CNS adverse events were reported: insomnia/sleep disturbances (26.9\%; 53/197); abnormal dreams $(18.8 \%$; 37/197); depression (17.3\%; 34/197); dizziness (15.2\%; 30/197); fatigue/tiredness (13.7\%; 27/197); and other reasons $(8.1 \% ; 16 / 197)$. Six months after the switch from EFV to RPV, 74.8\% (92/123) of patients reported an improvement of CNS symptoms, $14.6 \%(18 / 123)$ reported a stable condition and 3.2\% (4/ 123) described worsening CNS side effects. Continuous improvement in CNS symptoms at M12 was reported for $78.3 \%$ of patients for whom we had data (72/92) while condition remained stable for $6.5 \%$ (6/92) of patients.

\section{Effectiveness and safety of the RPV/TDF/FTC co- formulation}

Effectiveness and safety variables over time are presented both for treatment-naïve and -experienced patients in Table 4. Viral suppression (HIV-RNA $<50$ copies/mL) was achieved among 93.8\%, 97.6\% and 100\% of the cART naive patients at M6, M12 and M24 respectively $(P<0.001)$. At M24, 13 patients in the cART experienced group did not meet the criteria for virological suppression, i.e. HIVRNA $<50 / \mathrm{mL}$. These 13 patients were switched mostly from a PI regimen $(46 \%, 6 / 13)$ and from a NNRTI regimen $(38.5 \%, 5 / 13)$. Genotype was available for the 6 patients failing with an HIV-RNA $\geq 200$ copies/mL: 3 patients had a mutation conferring resistance to RPV at the time of virological failure, either $138 \mathrm{~A} / \mathrm{K}, 188 \mathrm{~L}$ or $221 \mathrm{Y}$ mutation. One of these 3 patients was not fully suppressed and already had developed a $188 \mathrm{~L}$ mutation at the time of the switch to 
Table 1 Demographic, clinical, immunological and virological baseline characteristics of patients initiating RPV/TDF/FTC co-formulation between April 1, 2013, and March 31, 2014

\begin{tabular}{|c|c|c|c|}
\hline Baseline characteristics & $\begin{array}{l}\text { Total } \\
(n=644)\end{array}$ & $\begin{array}{l}\text { cART-naïve patients } \\
(n=48)\end{array}$ & $\begin{array}{l}\text { CART-experienced } \\
\text { patients } \\
(n=596)\end{array}$ \\
\hline Mean age, years $( \pm S D$, median) & $45.8( \pm 11.1,46)$ & $42.3( \pm 11.3,44.5)$ & $46.1( \pm 11.0,46)$ \\
\hline Male gender, n (\%) & $451(70.0)$ & $41(85.4)$ & $410(68.8)$ \\
\hline \multicolumn{4}{|l|}{ HIV transmission group, $\mathrm{n}(\%)$} \\
\hline MSM & $307(47.7)$ & $29(60.4)$ & $278(46.6)$ \\
\hline Heterosexual & $260(40.4)$ & $14(29.2)$ & $246(41.3)$ \\
\hline Intravenous drug use & $44(6.8)$ & $2(4.2)$ & $42(7.1)$ \\
\hline Other $^{a}$ & $14(2.2)$ & $2(4.2)$ & $12(2.0)$ \\
\hline Unknown & $19(2.9)$ & $1(2.0)$ & $18(3.0)$ \\
\hline \multicolumn{4}{|l|}{ Ethnicity, n (\%) } \\
\hline White & $476(73.9)$ & $37(77.1)$ & $439(73.7)$ \\
\hline Black & $129(20.0)$ & $6(12.5)$ & $123(20.6)$ \\
\hline Hispano-American & $18(2.8)$ & $4(8.3)$ & $14(2.3)$ \\
\hline Asian & $20(3.1)$ & $1(2.1)$ & $19(3.2)$ \\
\hline Other & $1(0.2)$ & $0(0)$ & $1(0.2)$ \\
\hline Mean duration of HIV infection in years ( $\pm \mathrm{SD}$, median) & $11.0( \pm 7.8,9.8)$ & $2.6( \pm 3.3,1.5)$ & $11.7( \pm 7.7,10.6)$ \\
\hline History of AIDS disease, n (\%) & $91(14.1)$ & $0(0)$ & $91(15.3)$ \\
\hline Mean baseline ${ }^{\mathrm{b}} \mathrm{CD} 4$ count, cells $/ \mathrm{mm}^{3}\left( \pm \mathrm{SD}\right.$, median$^{c}$ & $637( \pm 271,606)$ & $478( \pm 176,473)$ & $650( \pm 273,620)$ \\
\hline Mean nadir CD4 count, cells $/ \mathrm{mm}^{3}( \pm \mathrm{SD} \text {, median) })^{\mathrm{d}}$ & $283( \pm 186,261.5)$ & $447( \pm 157,430)$ & $270( \pm 182,247)$ \\
\hline Baseline $^{\mathrm{b}}$ HIV-RNA $<50$ copies $/ \mathrm{mL}, \mathrm{n}(\%)^{c}$ & $552(86.1)$ & $2(4.2)^{e}$ & $549(92.6)$ \\
\hline HBV co-infection (positive AgHBs), n (\%) & $32(5.0)$ & $0(0)$ & $32(5.4)$ \\
\hline HCV co-infection (positive HCV-RNA), n (\%) & $35(5.4)$ & $1(2.1)$ & $34(5.7)$ \\
\hline
\end{tabular}

${ }^{\mathrm{a}}$ Other = blood products, perinatal transmission, other

${ }^{\mathrm{b}}$ Baseline $=$ at time of initiation of or switch to RPV/TDF/FTC co-formulation

'Missing data ( $n=641,48 / 48$ available in naïve, 593/596 in experienced)

${ }^{\mathrm{d}}$ Missing data ( $n=640,47 / 48$ available in naïve, 593/596 in experienced)

'Two CART-naïve patients started treatment with an HIV-RNA $<50$ copies/mL: one because of anxiety related to HIV-infection, the other was taking a combination

of tenofovir and emtricitabine intermittently without his physician knowledge. We still considered the latest as treatment-naïve of cART regimen

CART combined antiretroviral treatment, MSM men who have sex with men, SD standard deviation, HBV hepatitis B virus, HCV hepatitis C virus

RPV. Figure 1 shows the number of cART naive and experienced patients discontinuating RPV/TDF/FTC combination for virological failure or other reasons.

CD4 count significantly increased at M6, at M12 and at M24 compared to baseline values in both cART naive and experienced patients (Table 4).

For safety, we found that creatinine values significantly increased across the 3 time-points, M6, M12 and M24, in cART-experienced patients $(P<0.001)$ but not in cARTnaive patients. However, the creatinine clearance (or eGFR) decreased over time in both groups. Total cholesterol, triglycerides and HDL-cholesterol significantly decreased over time among cART-experienced patients (Table 4). When considering the subgroup of patients on PI-based regimen before the switch to RPV/TDF/FTC, cholesterol decreased significantly at M6 (mean 4.64 $\pm 0.96 \mathrm{SD}$, median 4.5) and M12 $(4.64 \pm 0.94,4.55)$ compared to baseline values $(5.09 \pm 0.98$ SD, median 5.01, $p<0.001)$. Similarly, HDLcholesterol decreased significantly at M6 (1.25 \pm 0.37 SD, median 1.17) and M12 (1.26 $\pm 0.38 \mathrm{SD}$, median 1.20) compared to baseline values $(1.31 \pm 0.40 \mathrm{SD}$, median 1.20 , $p=0.004)$. Triglycerides decreased significantly across time (M6: $1.41 \pm 1.01 \mathrm{SD}$, median 1.20; M12: $1.40 \pm 1.07$ $\mathrm{SD}$, median 1.12) compared to baseline $(1.73 \pm 1.21 \mathrm{SD}$, median $1.50, p<0.001)$ but at M24 values were not significantly different from those at baseline $(1.50 \pm 1.20$, $1.10, p=0.516)$.

\section{Follow-up on October 30, 2015}

On October 30, 2015, we assessed all patients who initiated a RPV/TDF/FTC co-formulation between March 2013 and April 2014. The median follow-up time since RPV/TDF/ FTC initiation was 18.4 months (interquartile range: 14.021.9). Overall, $166 / 644$ (25.8\%) patients had discontinued the RPV/TDF/FTC co-formulation on October 30, 2015 (Table 5).

Besides treatment failure as described above, the most common reasons provided for discontinuation were 
Table 2 Previous regimens at time of switch and main reasons for switch among the 596 cART-experienced patients initiating a RPV/TDF/FTC co-formulation

\begin{tabular}{ll}
\hline & $N(\%)$ \\
\hline ART regimen at switch & \\
2 NRTIs + EFV & $192(32.2)$ \\
2 NRTIs + NVP or ETV & $93(15.6)$ \\
2 NRTIs +1 PI & $156(26.2)$ \\
2 NRTIs +1 INSTI & $58(9.7)$ \\
Triple nuke regimen & $44(7.4)$ \\
Other & $29(4.9)$ \\
Unknown & $24(4.0)$ \\
Main reasons for switch & \\
Simplification & $266(44.6)$ \\
CNS toxicity & $143(24.0)$ \\
Physician decision & $46(7.7)$ \\
Gatrointestinal/liver toxicity & $42(7.0)$ \\
Abnormal fat distribution/dyslipidemia/concern & $38(6.4)$ \\
of cardiovascular disease & \\
Other toxicities (including endocrine, haematological, & $26(4.4)$ \\
kidney, muscle, skin) & \\
Patient wish/decision & $20(3.4)$ \\
Drug interaction & $6(1.0)$ \\
Treatment failure & $1(0.2)$ \\
Unknown & $8(1.3)$ \\
\hline
\end{tabular}

RPV/TDF/FTC rilpivirine/tenofovir/emtricitabine, cART combined antiretroviral treatment, NRTI nucleoside reverse transcriptase inhibitor, NNRTI non-nucleoside reverse transcriptase inhibitor, EFV efavirenz, NVP nevirapine, ETV etravirine, PI protease inhibitor, INSTI integrase strand transfer inhibitor

unknown (62/644; 9.6\% of all patients); physician decision with no reason specified $(34 / 644 ; 5.3 \%)$; patient request $(12 / 644 ; 1.9 \%)$; and CNS toxicity $(10 / 644 ; 1.6 \%)$. Among the 10 patients who discontinued RPV/TDF/ FTC co-formulation for CNS side effects, all were cART-experienced patients with 3 of them previously receiving EFV and who had already switched to RPV/ TDF/FTC for the same reason. There were no deaths among the study population. Of the 166 discontinuations, more than half $(n=92 ; 55.4 \%)$ had discontinued the RPV/TDF/FTC co-formulation for an integrase inhibitor-based regimen. Of these switches, $44.9 \%$ (45/ 92) switched to co-formulations including dolutegravir or elvitegravir.

\section{Discussion}

We demonstrated that rilpivirine, tenofovir disoproxil and emtricitabine in a fixed dose combination is an effective cART regimen in both treatment-experienced and -naïve patients under routine clinical conditions, although RPV was rarely used in naïve patients. Ninety-six percent of treatment-experienced patients and $100 \%$ of
Table 3 Central nervous system (CNS) adverse events experienced by the 123 patients on EFV-based regimens reporting CNS symptoms prior to switch and change over time on RPV/TDF/ FTC co-formulation

\begin{tabular}{ll}
\hline & $N(\%)$ \\
\hline CNS adverse events on previous & $197(74$ patients \\
EFV-based regimens & with > 1 symptoms) \\
Insomnia/sleep disturbances & $53(26.9 \%)$ \\
Abnormal dreams & $37(18.8 \%)$ \\
Symptoms of depression & $34(17.3 \%)$ \\
Dizziness/vertigo & $30(15.2 \%)$ \\
Fatigue/tiredness & $27(13.7 \%)$ \\
Other & $16(8.1 \%)$ \\
Change in CNS symptoms reported & 123 \\
by patients at 6 months after switch & \\
from EFV to RPV & \\
Worsening condition & $4(3.3 \%)$ \\
Stable condition & $18(14.6 \%)$ \\
Improved condition & $92(74.8 \%)$ \\
Other/unknown & $9(7.3 \%)$ \\
Further change in CNS symptoms at & 92 \\
12 months after switch from EFV to & \\
RPV in those who improved at M6 & \\
Worsening condition & $13(14.1 \%)$ \\
Stable condition & $1(1.1 \%)$ \\
Improved condition & $6(6.5 \%)$ \\
Other/unknown & $72(78.3 \%)$ \\
\hline CNS central nervous system, EFV efavirenz, RPV rilpivirine & M6 month 6
\end{tabular}

treatment-naïve patients were virologically suppressed at M24. Our findings are similar to clinical trials that showed $84-86 \%$ of virological success for RPV/TDF/FTC at M12 $[3,4]$ and $84 \%$ at M24 [23] in naïve patients, and $85.8 \%$ of virological success for RPV at M12 in experienced patients [5]. Our results are also consistent with observational studies assessing a treatment switch to RPV ( $>93 \%$ of virological success of RPV at M12) [6, 7], but none of them assessed the use of RPV among naïve patients under routine clinical care. Only one observational study reported a lower proportion of virological suppression at M12; results were explained by an inappropriate switch to a RPV-containing regimen in patients with previous virological failure and by missing data [11]. In our study, 13 treatment-experienced patients had an HIV-RNA above 50 copies/ $\mathrm{ml}$ at $\mathrm{M} 24$. We were able to obtain genotypic data in the 6 patients failing with an HIV-RNA $\geq 200$ copies/mL: 3 patients carried $138 \mathrm{~A} / \mathrm{K}, 188 \mathrm{~L}$ or $221 \mathrm{Y}$ mutations at the time of failure, mutations that are known to confer resistance to RPV [24]. One patient had a detectable viral load and had already developed a $188 \mathrm{~L}$ mutation at the time of switch and should have not received the RPV/TDF/FTC combination. The rate of confirmed treatment failure was therefore very low in our study and 
Table 4 Efficacy and safety parameters (mean values \pm standard deviation, median) at M6, M12 and M24 after initiation (baseline) of a RPV/TDF/FTC co-formulation among cART-naive and CART-experienced patients

\begin{tabular}{|c|c|c|c|c|c|}
\hline & Baseline $^{t}$ & $M 6^{t+}$ & $M 12^{t+t}$ & $M 24^{t+t t}$ & $P$ value over time* \\
\hline \multicolumn{6}{|c|}{ Viral load (copies/mL), n (\%) } \\
\hline \multicolumn{6}{|l|}{ cART-naïve } \\
\hline$<50$ & $2(4.2)^{*}$ & $45(93.8)$ & $41(97.6)$ & $26(100)$ & \multirow[t]{2}{*}{$<0.001$} \\
\hline$>=50$ & $46(95.8)$ & $3(6.2)$ & $1(2.4)$ & $0(0)$ & \\
\hline \multicolumn{6}{|l|}{ CART-experienced } \\
\hline$<50$ & $549(92.6)$ & $562(96.2)$ & $514(97.5)$ & $380(96.7)$ & \multirow[t]{2}{*}{0.002} \\
\hline$>=50$ & $44(7.4)$ & $22(3.8)$ & $13(2.5)$ & $13(3.3)$ & \\
\hline \multicolumn{6}{|c|}{$\mathrm{CD} 4$ count $\left(\right.$ cells $\left./ \mathrm{mm}^{3}\right), \pm \mathrm{SD}$, median } \\
\hline CART-naïve & $478( \pm 176,473)$ & $622( \pm 204,602)$ & $621( \pm 201,581)$ & $704( \pm 248,649)$ & 0.004 \\
\hline CART-experienced & $650( \pm 273,620)$ & $676( \pm 285,643)$ & $681( \pm 286,633)$ & $697( \pm 363,660)$ & 0.001 \\
\hline \multicolumn{6}{|c|}{ ALAT (UI/L), $\pm S D$, median } \\
\hline cART-naïve & $31( \pm 18,28)$ & $31( \pm 16,30)$ & $31( \pm 16,28)$ & $30( \pm 14,32)$ & 0.578 \\
\hline cART-experienced & $39( \pm 49,29)$ & $38( \pm 42,29)$ & $35( \pm 25,29)$ & $36( \pm 63,28)$ & 0.284 \\
\hline \multicolumn{6}{|c|}{ Creatinine $(\mu \mathrm{mmol} / \mathrm{L}), \pm \mathrm{SD}$, median } \\
\hline cART-naïve & $94( \pm 82,82)$ & $88( \pm 15,86)$ & $88( \pm 15,86)$ & $91( \pm 13,89)$ & 0.887 \\
\hline cART-experienced & $78( \pm 16,77)$ & $85( \pm 18,85)$ & $86( \pm 18,86)$ & $85( \pm 21,83)$ & $<0.001$ \\
\hline \multicolumn{6}{|c|}{$\mathrm{eGFR}\left(\mathrm{mL} / \mathrm{min} / 1.73 \mathrm{~m}^{2}\right), \pm \mathrm{SD}$, median } \\
\hline cART-naïve & $98( \pm 25,100)$ & $90( \pm 20,89)$ & $91( \pm 21,91)$ & $90( \pm 26,86)$ & 0.008 \\
\hline CART-experienced & $100( \pm 25,96)$ & $90( \pm 28,86)$ & $88( \pm 23,85)$ & $89( \pm 26,87)$ & $<0.001$ \\
\hline \multicolumn{6}{|c|}{ Cholesterol (mmol/L), $\pm \mathrm{SD}$, median } \\
\hline cART-naïve & $4.7( \pm 1.0,4.8)$ & $4.5( \pm 1.0,4.4)$ & $4.5( \pm 1.0,4.4)$ & NA & 0.270 \\
\hline cART-experienced & $5.1( \pm 1.0,5.0)$ & $4.7( \pm 1.0,4.6)$ & $4.7(1.0,4.6)$ & NA & $<0.001$ \\
\hline \multicolumn{6}{|c|}{ Triglycerides (mmol/L), $\pm \mathrm{SD}$, median } \\
\hline CART-naïve & $1.5( \pm 1.1,1.2)$ & $1.5( \pm 1.1,1.3)$ & $1.5( \pm 0.9,1.4)$ & $1.3( \pm 0.6,1.2)$ & 0.347 \\
\hline cART-experienced & $1.6( \pm 1.3,1.3)$ & $1.4( \pm 0.9,1.2)$ & $1.4( \pm 0.9,1.2)$ & $1.4( \pm 0.9,1.2)$ & $<0.001$ \\
\hline \multicolumn{6}{|c|}{$\mathrm{HDL}$-cholesterol (mmol/L), $\pm \mathrm{SD}$, median } \\
\hline CART-naïve & $1.2( \pm 0.4,1.2)$ & $1.2( \pm 0.3,1.2)$ & $1.1( \pm 0.3,1.2)$ & NA & 0.195 \\
\hline CART-experienced & $1.3( \pm 0.4,1.3)$ & $1.3( \pm 0.3,1.2)$ & $1.2( \pm 0.3,1.2)$ & NA & $<0.001$ \\
\hline \multicolumn{6}{|c|}{ BMI $\left(\mathrm{kg} / \mathrm{m}^{2}\right), \pm \mathrm{SD}$, median } \\
\hline CART-naïve & $23.6( \pm 5.6,22.6)$ & $24.2( \pm 3.8,23.7)$ & $24.2( \pm 3.9,23.8)$ & $22.9( \pm 1.0,22.9)$ & 0.006 \\
\hline cART-experienced & $24.9( \pm 5.3,24.4)$ & $24.7( \pm 4.0,24.3)$ & $24.7( \pm 3.9,24.3)$ & $24.8( \pm 4.6,24.0)$ & 0.096 \\
\hline
\end{tabular}

${ }^{\mp}$ Missing data (ASAT 37/48 available in naïve patients, 565/596 in experienced; ALAT 37/48 available in naïve patients, 576/596 in experienced; creatinine and eGFR 33/48 available in naïve patients, 463/596 in experienced; triglycerides 36/48 available in naïve patients, 540/596 in experienced; cholesterol 36/48 available in naïve patients, 542/596 in experienced; HDL 36/48 available in naïve patients, 530/596 in experienced; weight 48/48 available in naïve patients, 574/596 in experienced; waist and hip 48/48 available in naïve patients, 563/596 in experienced; BMI 48/48 available in naïve patients, $574 / 596$ in experienced)

${ }^{++}$Missing data (ASAT/ALAT/Cholesterol 48/48 available in naïve patients, 583/596 in experienced; creatinine/eGFR/triglycerides 48/48 available in naïve patients, 578/596 in experienced; HDL/weight/BMI 48/48 available in naïve patients, 582/596 in experienced; waist and hip 48/48 available in naïve patients, 580/596 in experienced)

${ }_{++1}^{+1}$ Missing data (ASAT/ALAT 42/48 available in naïve patients, 527/596 in experienced; creatinine 42/48 available in naïve patients, 521/596 in experienced; eGFR $41 / 48$ available in naïve patients, 517/596 in experienced; triglycerides $41 / 48$ available in naïve patients, 522/596 in experienced; cholesterol 41/48 available in naïve patients, 526/596 in experienced; HDL 41/48 available in naïve patients, 525/596 in experienced; weight 44/48 available in naïve patients, 513/596 in experienced; waist $44 / 48$ available in naïve patients, 511/596 in experienced; hip 44/48 available in naïve patients, 510/596 in experienced; BMI 44/48 available in naïve patients, 513/596 in experienced)

${ }^{++t+}$ Missing data (ALAT 15/48 available in naïve patients, 239/596 in experienced; creatinine 14/48 available in naïve patients, 236/596 in experienced; eGFR $14 / 48$ available in naïve patients, $235 / 596$ in experienced; triglycerides $14 / 48$ available in naïve patients, 232/596 in experienced; cholesterol 0/48 available in naïve patients, $0 / 596$ in experienced; HDL 0/48 available in naive patients, $0 / 596$ in experienced; weight 4/48 available in naïve patients, 158/596 in experienced; waist 4/48 available in naïve patients, 151/596 in experienced; hip 4/48 available in naïve patients, 151/596 in experienced; BMI 4/48 available in naïve patients, $158 / 596$ in experienced)

${ }^{*} P$-values from generalized estimating equation (for viral load) and linear multilevel models (CD4, ALAT, creatinine, eGFR, cholesterol, triglycerides, HDL-cholesterol and BMI) comparing data at M6, M12 and M24 to baseline among CART-naïve and CART-experienced patients separately, after adjustment for main confounders

RPV/TDF/FTC rilpivirine/tenofovir/emtricitabine, CART combined antiretroviral treatment, ASAT aspartate aminotransferase, ALAT alanine aminotransferase, eGFR glomerular filtration rate, HDL high-density lipoprotein, BMI body mass index

${ }^{*}$ Two cART-naïve patients started treatment with an HIV-RNA $<50$ copies $/ \mathrm{mL}$ : one because of anxiety related to HIV-infection, the other was taking a combination of tenofovir and emtricitabine intermittently without his physician knowledge 


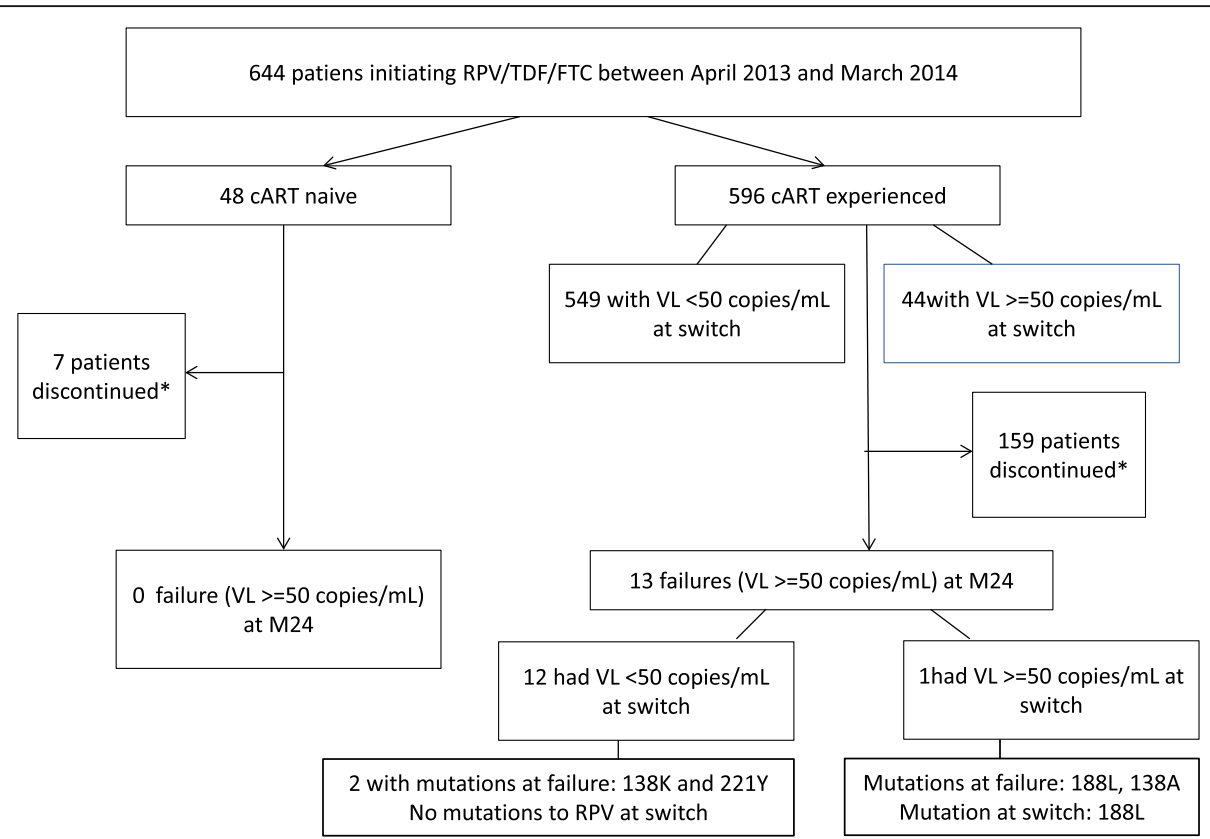

Fig. 1 Flowchart of patients initiating RPV/TDF/FTC co-formulation, discontinuation and treatment failures. *Discontinuation for other reasons than virological failure VI viral load, CART combined antiretroviral therapy

detectable HIV-RNA may translate blips in viral load or poor adherence rather than virological failure.

Among cART-experienced patients, the main reasons for a switch to the newly marketed RPV/TDF/FTC coformulation were treatment simplification and CNS toxicity. The proportion of patients switching to RPV for simplification is slightly lower than in other observational studies $[6,11]$. Unlike previous reports $[6,7,11]$, we accurately assessed the reasons for a switch to RPV with a dedicated detailed questionnaire. Due to the retrospective manner and a non-response rate of $9.5 \%$ to our standardized questionnaire, it is possible that the simplification reason was underreported or rather stated as "physician decision" or "patient request". Among those who switched because of CNS toxicity, most were on an EFV-based regimen. Symptoms did improve in approximately $75 \%$ of patients 6 months after the switch with a continuous improvement at M12. Moreover, only 3 patients switched from EFV to RPV/TDF/FTC later discontinued RPV coformulation for persisting CNS side effects. While there was no change in neurocognitive functions after EFV replacement for a PI drug in a small controlled trial enrolling 16 patients [25], another observational study confirmed improvement in neurological side-effects in almost $50 \%$ of patients switching from an EFV-based regimen to RPV [6]. Switch from EFV to RPV seems therefore a reasonable and sustainable option for patients experiencing CNS side effects on EFV.

Overall, the RPV/TDF/FTC co-formulation was safe and well tolerated in most patients. As shown in previous studies $[6,7,11]$, we observed a significant increase in creatinine over time among our study population, but this difference was not clinically relevant. Rilpivirine is known to inhibit the creatinine transporter in the proximal renal tubule [13]. Similarly to other studies $[6,7,11]$, we did observe a significant change in the lipid profile of treatmentexperienced patients after the switch to RPV/TDF/FTC. This was also true for the subset of patients previously on PI. However, there was no effect of RPV/TDF/FTC on lipid profile over time among cART-naïve patients; this may be due to the small number of treatment naïve subjects included and the lack of power to detect an effect.

Despite good results for the effectiveness and tolerability of the RPV/TDF/FTC co-formulation, a quarter of patients changed their ART regimen, mainly upon physician decision, after a mean time of 18.4 months. This is much shorter than the reported mean duration of patients on first- and second-line newer cART regimens (4.6 and 3.9 years, respectively) in the 2008-2011 period as described in the HIV Outpatient Study [26]. We hypothesized that there is a low confidence in the RPV genetic barrier among treating physicians [27], particularly when compared to newer once-daily, inhibitor-based co-formulation regimens, the latest being marketed in Switzerland only 2 years after the RPV/TDF/FTC co-formulation. The need for a fatty meal intake may be also a barrier to the large-scale prescription of this regimen although the acceptability of food constraint was not formally assessed in our study. Finally, less than $5 \%$ of patients discontinued the RPV/ TDF/FTC co-formulation due to side-effects, mainly CNS- 
Table 5 CART-experienced and CART-naive RPV/TDF/FTC patients who discontinued treatment between April 2014 and October 2015; reasons for discontinuation and next CART regimen

\begin{tabular}{|c|c|c|}
\hline & \multicolumn{2}{|c|}{ Number (\%) of patients } \\
\hline & $\begin{array}{l}\text { cART-naïve } \\
\text { RPV/TDF/FTC } \\
(n=48)\end{array}$ & $\begin{array}{l}\text { cART- } \\
\text { experienced } \\
\text { RPV/TDF/FTC } \\
(n=596) \\
\end{array}$ \\
\hline Number of patients discontinuating & $7(14.5 \%)$ & $159(26.7 \%)$ \\
\hline \multicolumn{3}{|l|}{ Reasons for discontinuation } \\
\hline Treatment failure & $1(2.1 \%)$ & $5(0.8 \%)$ \\
\hline Physician decision & $2(4.2 \%)$ & $34(5.7 \%)$ \\
\hline Patient request & $1(2.1 \%)$ & $11(1.8 \%)$ \\
\hline CNS toxicity & $0(0 \%)$ & $10(1.7 \%)$ \\
\hline Availability of more effective treatment & $1(2.1 \%)$ & $7(1.2 \%)$ \\
\hline Kidney toxicity & $0(0 \%)$ & $7(1.2 \%)$ \\
\hline $\begin{array}{l}\text { Other toxicities (including } \\
\text { gastrointestinal, liver, endocrine, } \\
\text { dyslipidemia, abnormal fat distribution) }\end{array}$ & $0(0 \%)$ & $8(1.3 \%)$ \\
\hline $\begin{array}{l}\text { Other causes (pregnancy, enrolment } \\
\text { in drug trial, drug interaction, prevention } \\
\text { of side-effects, non-compliance) }\end{array}$ & $0(0 \%)$ & $8(1.3 \%)$ \\
\hline Unspecified causes & $0(0 \%)$ & $9(1.5 \%)$ \\
\hline Unknown & $2(4.2 \%)$ & $60(10.1 \%)$ \\
\hline \multicolumn{3}{|l|}{ Next treatment regimen after discontinuation } \\
\hline \multicolumn{3}{|l|}{ INSTI-based } \\
\hline DTG/ABC/3TC co-formulation & $2(4.2 \%)$ & $25(4.2 \%)$ \\
\hline EGV/COB/TDF/FTC co-formulation & $2(4.2 \%)$ & $16(2.7 \%)$ \\
\hline Other DTG-based regimen & $1(2.1 \%)$ & $29(4.9 \%)$ \\
\hline Other EVG-based regimen & $0(0 \%)$ & $3(0.5 \%)$ \\
\hline RAL-based regimen & 1 (1.2\%) & $13(2.2 \%)$ \\
\hline PI-based & $0(0 \%)$ & $18(3.0 \%)$ \\
\hline NNRTI-based & $0(0 \%)$ & $24(4.0 \%)$ \\
\hline Other & $0(0 \%)$ & $4(0.7 \%)$ \\
\hline Unknown & $1(1.2 \%)$ & 27 (4.5\%) \\
\hline
\end{tabular}

CART combined antiretroviral treatment, RPV/TDF/FTC rilpivirine/tenofovir/ emtricitabine, CNS central nervous system, INSTI integrase strand transfer inhibitor, DTG dolutegravir, ABC abacavir, 3TC emtricitabine, EGV elvitegravir, $\mathrm{COB}$ cobicistat, PI protease inhibitor

related symptoms and kidney toxicity. Neuropsychiatric adverse events due to RPV were described in up to $27 \%$ of naïve patients receiving 48 weeks of RPV in clinical trials, but discontinuation was rare [16]. In our study, discontinuation of RPV for CNS toxicity (1.6\%) was much lower than discontinuation of EFV for the same reason (65.6\%). It is also lower than discontinuation rates reported on dolutegravir in routine clinical settings, which ranged between $3.4 \%$ and $6 \%$ [28].

Although we included a small number of cART-naïve patients, our study is the first to report on treatment outcomes up to 24 months under routine clinical conditions in this population. This may provide the necessary confidence to clinicians to prescribe the RPV/TDF/FTC co-formulation to HIV-naïve patients if the virological criteria are met, and providing that the patient consents to comply with the dietary restrictions.

Our study has several limitations. First, the standardized questionnaire on the reasons for a switch to the RPV/TDF/ FTC co-formulation was retrospective and therefore subject to recall bias, particularly regarding the possible overreporting of CNS toxicity symptoms and their improvement after a switch from EFV to RPV. Second, the study population was mainly Caucasian, male, and MSM, which renders more difficult the generalizability of our results to under-represented transmission groups. Finally, we did not compare initiation to RPV/TDC/FTC co-formulation to starts and switches to alternative regimens during the same time period.

\section{Conclusions}

The use of RPV is safe and effective under routine clinical conditions, both in a switch strategy and in naïve patients. Our study supports policy changes made in Switzerland in October 2014 to use RPV for cART-experienced virologically suppressed patients. RPV demonstrates a favourable neurological toxicity profile with most patients experiencing CNS side effects on EFV improving after a switch to RPV and very low discontinuation rate for CNS events when compared to EFV or integrase inhibitor. Future research should focus on comparing the RPV/TDF/FTC co-formulation with newer available regimens containing integrase inhibitors in a switch strategy, including an assessment of cost-effectiveness. The reasons for early discontinuation of RPV/TDF/FTC co-formulation based on physician decision in the SHCS should also be further explored.

\section{Abbreviations}

AIDS: Acquired immunodeficiency syndrome; CART: Combined antiretroviral therapy; CNS: Central nervous system; EFV: Efavirenz; eGRF: Estimated glomerular function rate; FTC: Emtricitabine; HBV: Hepatitis B virus; HCV: Hepatitis C virus; HICDEP: HIV Cohorts Data Exchange Protocol; HIV: Human immunodeficiency virus; M12: Month 12; M24: Month 24; M6: Month 6; MSM: Men who have sex with men; RPV: Rilpivirine; SHCS: Swiss HIV cohort study; TDF: Tenofovir

\section{Acknowledgments}

We would like to thank Rickenbach $M$ and Schöni-Affolter $F$ for retrieving data from the SHCS database, and all members of the Swiss HIV Cohort Study (SHCS): Aubert V, Battegay M, Bernasconi E, Böni J, Bucher HC, Burton-Jeangros C, Calmy A, Cavassini M, Dollenmaier G, Egger M, Elzi L, Fehr J, Fellay J, Furrer H (Chairman, Clinical and Laboratory Committee), Fux CA, Gorgievski M, Günthard H (President, SHCS), Haerry D (deputy of "Positive Council"), Hasse B, Hirsch HH, Hoffmann M, Hösli I, Kahlert C, Kaiser L, Keiser O, Klimkait T, Kouyos R, Kovari H, Ledergerber B, Martinetti G, Martinez de Tejada B, Metzner K, Müller N, Nadal D, Nicca D, Pantaleo G, Rauch A (Chairman, Scientific Board), Regenass S, Rickenbach M (Head, Data Centre), Rudin C (Chairman, Mother \& Child Substudy), Schöni-Affolter F, Schmid P, Schüpbach J, Speck R, Tarr P, Telenti A, Trkola A, Vernazza P, Weber R, Yerly S. The data are gathered by the 5 Swiss university hospitals, 2 cantonal hospitals, 15 affiliated hospitals, and 36 private physicians (lhttp://www.shcs.ch/180-health-care-providers). 


\section{Funding}

The study was sponsored by the Swiss HIV Cohort Study with a grant from Gilead Sciences.

\section{Availability of data and materials}

The datasets used and analysed during the current study are available from the corresponding author on reasonable request.

\section{Authors' contributions}

$D S, A C, B E$ and AGA conceived the study and the analyses. DS, AC and BE conducted reviews to inform model parameters. AGA conducted the analyses. DS wrote the manuscript. JF, CH, PS, MB, MC, EB and AC contributed to the acquisition of data. JF, PS, MB and MC also made substantial contributions to the conception and design of the study protocol. All authors contributed to the revision of the manuscript for important intellectual content.

\section{Ethics approval and consent to participate}

The Swiss HIV Cohort Study has been approved by the "Commission Cantonale d'Ethique de la Recherche de Genève" which belongs to the "Département de l'emploi, des affaires sociales et de la santé de la République et Canton de Genève". This prospective analysis was done using SHCS data and was approved by the scientific board of the SHCS on July 2014. All patients signed the informed consent form before enrolment in the SHCS.

\section{Consent for publication}

Not applicable.

\section{Competing interests}

The study was sponsored by the SHCS with a grant from Gilead Sciences. Gilead Sciences had no role in data collection, in data analysis, or interpretation. D.S. has participated to one advisory board meeting for Gilead Sciences. A.C. received an institutional unrestricted educational grant from Gilead Sciences to support the funding of activities in the Geneva area.

\section{Publisher's Note}

Springer Nature remains neutral with regard to jurisdictional claims in published maps and institutional affiliations.

\section{Author details}

'Division of Infectious Diseases, University Hospital Geneva, Geneva, Switzerland. ${ }^{2}$ Clinical Research Center and Division of Clinical Epidemiology, Department of Health and Community Medicine, University Hospital Geneva, Geneva, Switzerland. ${ }^{3}$ Division of Infectious Diseases and Hospital Epidemiology, University Hospital Basel, Basel, Switzerland. ${ }^{4}$ Division of Infectious Diseases, University Hospital of Lausanne, Lausanne, Switzerland. ${ }^{5}$ Division of Infectious Diseases and Hospital Epidemiology, University Hospital Zurich, University of Zurich, Zurich, Switzerland. ${ }^{6}$ University Clinic of Infectious Diseases, University Hospital Bern, Bern, Switzerland. 'Division of Infectious Diseases and Hospital Epidemiology, St. Gallen Cantonal Hospital, St. Gallen, Switzerland. ${ }^{8}$ Division of Infectious Diseases, Ospedale Regionale, Lugano, Switzerland.

Received: 23 December 2016 Accepted: 29 June 2017

Published online: 06 July 2017

\section{References}

1. Cohen CJ, Andrade-Villanueva J, Clotet B, et al. Rilpivirine versus efavirenz with two background nucleoside or nucleotide reverse transcriptase inhibitors in treatment-naive adults infected with HIV-1 (THRIVE): a phase 3, randomised, non-inferiority trial. Lancet. Jul 16 2011;378(9787):229-237.

2. Molina JM, Cahn P, Grinsztejn B, et al. Rilpivirine versus efavirenz with tenofovir and emtricitabine in treatment-naive adults infected with HIV-1 (ECHO): a phase 3 randomised double-blind active-controlled trial. Lancet. Jul 16 2011;378(9787):238-246.

3. Cohen C, Wohl D, Arribas JR, et al. Week 48 results from a randomized clinical trial of rilpivirine/emtricitabine/tenofovir disoproxil fumarate vs. efavirenz/emtricitabine/tenofovir disoproxil fumarate in treatment-naive HIV1-infected adults. AIDS. Apr 24 2014;28(7):989-997.

4. Cohen CJ, Molina JM, Cahn P, et al. Efficacy and safety of rilpivirine (TMC278) versus efavirenz at 48 weeks in treatment-naive HIV-1-infected patients: pooled results from the phase 3 double-blind randomized ECHO and THRIVE Trials. J Acquir Immune Defic Syndr. May 1 2012;60(1):33-42.

5. Palella FJ, Jr., Fisher M, Tebas $P$, et al. Simplification to rilpivirine/emtricitabine/ tenofovir disoproxil fumarate from ritonavir-boosted protease inhibitor antiretroviral therapy in a randomized trial of HIV-1 RNA-suppressed participants. AIDS. Jan 28 2014:28(3):335-344.

6. Cazanave C, Reigadas S, Mazubert C, et al. Switch to Rilpivirine/Emtricitabine/ Tenofovir Single-Tablet Regimen of Human Immunodeficiency Virus-1 RNASuppressed Patients, Agence Nationale de Recherches sur le SIDA et les Hepatites Virales CO3 Aquitaine Cohort, 2012-2014. Open Forum Infect Dis. Jan 2015;2(1):ofv018.

7. Pinnetti C, Di Giambenedetto S, Maggiolo F, et al. Switching to Coformulated Rilpivirine/Emtricitabine/Tenofovir in Virologically Suppressed Patients: Data From a Multicenter Cohort. J Acquir Immune Defic Syndr. Dec 1 2015;70(4):e147-e150

8. Mills AM, Cohen C, Dejesus E, et al. Efficacy and safety 48 weeks after switching from efavirenz to rilpivirine using emtricitabine/tenofovir disoproxil fumaratebased single-tablet regimens. HIV Clin Trials. Sep-Oct 2013;14(5):216-23.

9. Allavena $C$, Dailly $E$, Reliquet $V$, et al. Switching from tenofovir/emtricitabine and nevirapine to a tenofovir/emtricitabine/rilpivirine single-tablet regimen in virologically suppressed, HIV-1-infected subjects. J Antimicrob Chemother. Oct 2014;69(10):2804-8.

10. Rokx C, Verbon A, Rijnders BJ. Successful switch to rilpivirine/tenofovir/ emtricitabine in HIV-1-infected patients with an isolated K103N mutation acquired during prior nonnucleoside reverse transcriptase inhibitor therapy. HIV Med. Nov 2014;15(10):611-4

11. Gazaignes S, Resche-Rigon M, Gatey C, et al. Efficacy and safety of a switch to rilpivirine-based regimens in treatment-experienced HIV-1-infected patients: a cohort study. Antivir Ther. 2016;21(4):329-36.

12. Schouten J, Wit FW, Stolte IG, et al. Cross-sectional comparison of the prevalence of age-associated comorbidities and their risk factors between HIV-infected and uninfected individuals: the AGEhIV cohort study. Clin Infect Dis. Dec 15 2014;59(12):1787-1797.

13. Imaz A, Podzamczer D. The role of rilpivirine in clinical practice: strengths and weaknesses of the new nonnucleoside reverse transcriptase inhibitor for HIV therapy. AIDS Rev. Oct-Dec 2012;14(4):268-78.

14. Molina JM, Clumeck N, Orkin C, Rimsky LT, Vanveggel S, Stevens M. Week 96 analysis of rilpivirine or efavirenz in HIV-1-infected patients with baseline viral load $</=100000$ copies/mL in the pooled ECHO and THRIVE phase 3, randomized, double-blind trials. HIV Med. Jan 2013:15(1):57-62.

15. Nelson MR, Elion RA, Cohen CJ, et al. Rilpivirine versus efavirenz in HIV-1infected subjects receiving emtricitabine/tenofovir DF: pooled 96-week data from ECHO and THRIVE studies. HIV Clin Trials. May-Jun 2013;14(3):81-91.

16. Mills AM, Antinori A, Clotet B, et al. Neurological and psychiatric tolerability of rilpivirine (TMC278) vs. efavirenz in treatment-naive, HIV-1-infected patients at 48 weeks. HIV Med. Aug 2013;14(7):391-400.

17. National Institute of Health. Guidelines for the USe of Antiretroviral Agents in HIV1-Infected Adults and Adolescents 2015. https:/aidsinfo.nih.gov/contentfiles/ Ivguidelines/AA Recommendations.pdf. Accessed 20 November 2015.

18. European AIDS Clinical Society. Guidelines Version 8.0 October 2015. 2015. http://www.eacsociety.org/files/2015_eacsguidelines_8.0-english_revised20151104.pdf. Accessed 20 November 2015.

19. Swiss HIV Cohort Study. about SHCS. http://www.shcs.ch/157-about-shcs. Accessed 20 November 2015.

20. Schoeni-Affolter F, Ledergerber B, Rickenbach M, et al. Cohort profile: the Swiss HIV cohort study. Int J Epidemiol. Oct 2010;39(5):1179-89.

21. Kohler P, Schmidt AJ, Cavassini M, et al. The HIV care cascade in Switzerland: reaching the UNAIDS/WHO targets for patients diagnosed with HIV. AIDS. 2015:29(18):2509-15

22. EuroCoord. HICDEP 1.60. 2012; http://www.hicdep.org/wiki/Hicdep_1.60. Accessed 01 December 2015.

23. Behrens $\mathrm{G}$, Rijnders $B$, Nelson M, et al. Rilpivirine versus efavirenz with emtricitabine/tenofovir disoproxil fumarate in treatment-naive HIV-1infected patients with HIV-1 RNA </=100,000 copies/mL: week 96 pooled ECHO/THRIVE subanalysis. AIDS Patient Care STDs. Apr 2014;28(4):168-75.

24. Sluis-Cremer N, Wainberg MA, Schinazi RF. Resistance to reverse transcriptase inhibitors used in the treatment and prevention of HIV-1 infection. Future Microbiol. 2015;10(11):1773-82.

25. Payne B, Chadwick TJ, Blamire A, et al. Does efavirenz replacement improve neurological function in treated HIV infection? HIV Med. 2017. doi:10.1111/ hiv. 12503 . 
26. Sheth AN, Ofotokun I, Buchacz K, et al. Antiretroviral Regimen Durability and Success in Treatment-Naive and Treatment-Experienced Patients by Year of Treatment Initiation, United States, 1996-2011. J Acquir Immune Defic Syndr. Jan 1 2016;71(1):47-56.

27. Porter DP, Kulkarni R, Fralich T, Miller MD, White KL. 96-week resistance analyses of the STaR study: rilpivirine/emtricitabine/tenofovir DF versus efavirenz/emtricitabine/tenofovir DF in antiretroviral-naive, HIV-1-infected subjects. HIV Clin Trials. Jan-Feb 2015;16(1):30-8.

28. Menard A, Montagnac C, Solas C, et al. Neuropsychiatric adverse effects on dolutegravir: an emerging concern in Europe. AIDS. May 15 2017;31(8): 1201-1203.

Submit your next manuscript to BioMed Central and we will help you at every step:

- We accept pre-submission inquiries

- Our selector tool helps you to find the most relevant journal

- We provide round the clock customer support

- Convenient online submission

- Thorough peer review

- Inclusion in PubMed and all major indexing services

- Maximum visibility for your research

Submit your manuscript at www.biomedcentral.com/submit 